\title{
Designing with Care: The Future of Pervasive Healthcare
}

\author{
Ingrid Mulder, Yvonne Schikhof, Martijn Vastenburg, Alan Card, Tory Dunn, \\ Andreas Komninos, Marilyn McGee-Lennon, Mark Santcroos, \\ Gabriele Tiotto, Mieke van Gils, Jan-Willem van 't Klooster, Annelies Veys, \\ and Mohammed Zarifi Eslami
}

T he first Designing with Care workshop (http://designingwithcare09. org) was organized in conjunction with the 3rd International Conference on Pervasive Computing Technology for Healthcare (PervasiveHealth 2009) in London. This workshop focused on human values in relation to the design and evaluation of pervasive technology for healthcare.

Today's healthcare technologies are increasingly pervasive, moving into healthcare professionals' working environment and patients' homes, presenting new challenges to designing and evaluating healthcare applications. Current approaches tend to be subjective, piecemeal, or both. Individual approaches to evaluation risk being incomplete, and comparisons between systems can be difficult.

The current debate on the design and evaluation of pervasive systems appeared in the April-June 2009 International Journal of Mobile Human Computer Interaction, a special issue on advances in evaluating mobile and ubiquitous systems. It shows that user participation in designing pervasive systems tends to be subjective, limited in time, and in an unrealistic setting, which results in findings with limited validity. Although these challenges can be addressed when designing perva- sive systems, designing and evaluating pervasive-healthcare applications in particular face additional challenges. These applications are increasingly perceived as useful for a diverse group of "nontypical" users, including older and disabled people.

\section{HUMANE CARE}

Humane care-which is an important design paradigm relevant for pervasive healthcare-means designing systems

\section{Understanding human values is essential to avoid dehumanizing and stigmatizing users.}

with care, keeping in mind both users and other stakeholders. Understanding human values is a key factor in creating any successful healthcare application and is essential to avoid dehumanizing and stigmatizing users. This is especially true for designing pervasive-healthcare applications, which are becoming increasingly sensitive to the complexity of users. By considering human values, designers and developers can create pervasivehealthcare applications that better fit users' needs and desires. Incorporat- ing human values into design can also reduce resistance to new technology by users and healthcare and social-care professionals.

Although much research has emphasized the need to ensure that healthcare technology is usable, appropriate, and acceptable for these groups, recognition of the importance of incorporating human values in the design is only now beginning. The often-referred-to UTAUT model ${ }^{1}$ on user acceptance and use of technology has been applied in healthcare, but the discussion of ethical issues is most often reserved for the point of implementation of new technologies rather than being paid attention to in the early phases of design.

\section{CURRENT PRACTICES}

Designing with Care 09 aimed to bring together researchers, designers, and practitioners to contribute to a vivid discussion, which would hopefully lead to improved methods and tools incorporating human values in the designs of pervasive-healthcare applications. One goal was to provide insights leading to a consistent set of methods and guidelines for designing and evaluating pervasive-healthcare applications. Participants were invited to share their experiences with incorporating values into the design of pervasive-healthcare 
systems and with understanding the sensitive issues involved in designing for healthcare.

The workshop had two parts: in the morning, participants presented best practices and cases (see the workshop's Web site for position statements and presentations). In the afternoon, participants discussed their experiences related to human values and healthcare application design. The discussion's purpose was to determine how to design with care and how to evaluate pervasive technology to assess human values and ethical issues in the context of use.

First, Alan Card (Evidence-Based Health Solutions) presented on pandemic flu. Those with chronic illness will almost certainly be at high risk for pandemic-flu complications, just as they are for seasonal flu. Although these patients require ongoing care to manage their conditions, the doctor's office might not be the safest place to receive it during a pandemic. Card argued that, on the basis of the principles of beneficence (do good) and nonmaleficence (do no harm), there's an ethical requirement to provide effective care that's also as safe as possible, even during a pandemic. He suggested that home telehealth technology could provide a way to meet these competing obligations. He discussed how several design considerations relate to the pandemic-flu scenario, including asynchronous versus synchronous communication, robustness, information density of communication (such as the amount of information conveyed per communication episode), and usability. The presentation also included an appreciation of how to balance these ethical imperatives and design constraints against user values and concerns such as usability, accessibility, privacy, intrusiveness, stigma, autonomy, the system's appropriateness, the usage environment, and language.

Next, Mieke van Gils (Katholieke Universiteit Leuven) reported on user acceptance of a social alarm system integrated into a larger communication platform. This platform uses audio and video communication to let elderly users make calls to friends and relatives and to make alarm calls to a centralized alarm center. Older users who live independently can stay in touch with the people they know. They might also feel safer, knowing they can contact the alarm center at any given time in case of emergency. Moreover, combining a communication system with a social alarm system could ease acceptance of the alarm system, because the system doesn't focus entirely on healthcarerelated issues.

Marilyn McGee-Lennon (University of Glasgow) presented the issues involved in capturing and satisfy-

User-centered design
for healthcare faces
additional problems
when involving
nontypical users.

ing multiple stakeholders' needs and requirements in the design of home care technology. Home care systems can range from simple standalone electromechanical alarms, such as to indicate a bath overflowing, to systems integrated into the home's physical infrastructure that monitor patient state, perform sophisticated analyses, deliver customized information to patients and clinicians, and support communication among them. The network of people involved in home care can be complex, ranging from the user or patient, to friends and family interested in their well-being, to various healthcare and social-care professionals interested in the information or results that the home care system provides.

With the number of stakeholders involved, it's crucial to adapt traditional user requirements methods or develop novel methods and tools for appropriately capturing the different stakeholders' needs, values, and goals.
It's also important to monitor these requirements and adapt them as a person's needs change. McGee-Lennon presented examples of user engagement work with a variety of stakeholders that explored the methods themselves, the individual stakeholder groups' needs and values, and possible conflicts in needs and values when multiple stakeholder groups are involved.

Andreas Komninos (Glasgow Caledonian University) focused on incidental mobile healthcare as part of a design background that aims to augment everyday mobile applications to provide healthcare. This would reduce the stigma for users of needing to employ "dedicated" devices or services. He discussed multimodal healthy "info-snacks" (snippets of information; the term was taken from the observed incidental information "snacking" behavior that mobile users exhibit ${ }^{2}$ ) as a mechanism for providing unobtrusive incidental health-oriented information and services via non-health-related applications serving as hosts. Komninos argued that healthy info-snacks should be provided implicitly and unobtrusively, flowing with, rather than disturbing, the naturally occurring pattern of interaction bursts that users display with their mobile devices.

Komninos discussed two prototype concepts. The first was a demonstration of how physical-activity info-snacks in a mobile tool for planning trips on public-transportation could help inform interface design and implicitly coerce users toward adopting more trips including increased physical activity. The second prototype examined implicit body interaction with music players on mobile devices. It created a fun challenge for users to help them achieve physical-activity-related targets (such as cardiac reconditioning or weight loss) during idle time (such as walking from the train station to work). The healthy info-snacking took the form of feedback on whether a user was meeting a walking-pace target: the music player increased or decreased the 
tempo of the user's music to reflect his or her performance.

Gabriele Tiotto (Politecnico di Torino) discussed design and implementation issues of a system that automatically translates Italian to Italian Sign Language through a virtual signing avatar. The system could be applicable to pervasive-healthcare systems as a platform for supporting communication between deaf patients and their doctors. Several surveys have shown how deaf people suffer great difficulties while accessing the Italian National Health Service, which also affects the quality of service provided to hearing people. So, this design of accessible applications for deaf people in healthcare is especially important. The system's approach incorporates accessibility and design-for-all values; a major issue is whether the deaf community will accept the system.

Jan-Willem van 't Klooster (University of Twente) discussed how he and his colleagues developed patientcentered telemedicine services, using patient interviews based on the International Classification of Functioning, Health, and Disability (ICF). The ICF is a classification for health in its biopsycho-social context; it incorporates biomedical, social, activity-related, personal, and external factors. Using this classification allows for holistic patient assessments and aids in the development of telemedicine care services; in van 't Klooster's work, this was an audience of people aged 60 and older. The interviews revealed the patients' complete health status, their daily activities, and both use of and wish for assistive technologies. Conducting such interviews is important not only from a user-centered design perspective but also for the ability to tailor and evolve such services. Van 't Klooster's work aims to design a services platform for integrated home care. This project includes collaboration with industrial and care partners, a scenario-based approach to user-need analysis, and implementation and tri- als of tailorable, evolvable telemedicine services.

Annelies Veys (Interdisciplinary Institute for Broadband Technology) argued that attention should be given to the strategic and organizational context when designing eCare services. From a systematic-thinking approach, Veys concluded that existing roles and actors from a care service's value network should be assessed in the design process. From the results of user and socio-economic studies, service designers should explore such value networks so as to delineate a financial model and a value proposition. Through scenarios, Veys and her

\section{We can learn much by applying best practices from human- centered design in the context of pervasive health.}

colleagues identified driving forces and bottlenecks.

Finally, Mohammed Zarifi Eslami (University of Twente) highlighted how to personalize the services provided by home healthcare systems according to individual patients' needs and preferences. Although some home healthcare applications have been proven in practice, many technological issues must be solved before home healthcare systems are cost-effective and efficient. However, he highlighted another category of issues, called architectural challenges, which stem from the fact that current home healthcare systems are generally standalone systems for specific diseases and assume a "standard" patient. But in reality, each patient is unique in how he or she experiences or is affected by a disease, because of not only his or her mental and physical condition but also his or her social and physical environment. So, it should be possible to personalize home healthcare systems' services according to individual patients' needs and preferences and to make incremental adaptations later if necessary.

\section{TOWARD A \\ RESEARCH AGENDA}

The challenges raised by the workshop participants provided fodder for a research agenda. Most of the problems encountered while designing for care were related to

- designing for specific target groups without ignoring differences in generations and education, or

- taking into account the new system's safety, reliability, accountability, and robustness while also aiming for labor-saving technology.

The workshop participants concluded that user-centered design for healthcare faces additional problems when involving nontypical users because they have diverse backgrounds, skills, living conditions, abilities, needs, and desires. Moreover, such nontypical users can be hard to reach or to involve in discussions.

Other issues on the research agenda were how to create sustainable solutions, how to provide fair access to the Internet for everyone, how to research social values, and which experiences can be reused from other sectors or countries. Participants all agreed that the effectiveness of the outcome is important, but that one has to take into account well-being and quality of life, participation in society, and ethical issues. For example, participants discussed how to address privacy in such a multistakeholder pervasivehealthcare context: Who's in control? Who should sign for informed consent?

Incorporating human values into the design process is in keeping with trends in human-centered design that go beyond usability and economic worth, and include moral discussions. In pervasive healthcare, however, traditional methods, such as ethnography, questionnaires, interviews, focus 
Ingrid Mulder is an associate professor of design techniques at the Delft University of Technology and heads the research center of Rotterdam University's School of Communication, Media, and Information Technology. Contact her at mulderi@acm.org.

Yvonne Schikhof is a lecturer at Rotterdam University's School of Health Care Studies. Contact her aty.schikhof@hro.nl.

Martijn Vastenburg is an assistant professor at Delft University of Technology's Faculty of Industrial Design Engineering. Contact him at m.h.vastenburg@tudelft.nl.

Alan Card is president and CEO of EvidenceBased Health Solutions. Contact him at ebhs.Ilc@ gmail.com.

groups, or observational research, predominate. Although "unconventional" methods, such as experience prototyping or an interaction theater using different scenarios, do capture human values, they're rarely used for designing pervasive-healthcare applications.

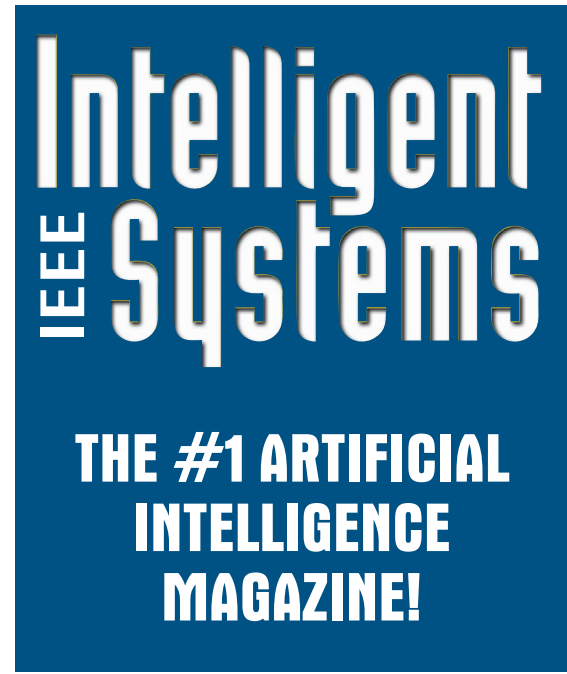

Tory Dunn is a user experience manager in Vodafone's UE group. Contact her at tory.dunn@ vodafone.com.

Andreas Komninos is a lecturer at Glasgow Caledonian University. Contact him at andreas. komninos@gcal.ac.uk.

Marilyn McGee-Lennon is a senior research fellow in the University of Glasgow's Department of Computing Science. Contact her at mcgeemr@dcs.gla.ac.uk.

Mark Santcroos is a lecturer at Rotterdam University's School of Communication, Media, and Information Technology. Contact him at m.a.santcroos@hro.nl.

Gabriele Tiotto is a PhD student at the Politecnico di Torino. Contact him at gabriele.tiotto@ polito.it.

W e can learn much by applying best practices from humancentered design in the context of pervasive health. In particular, design techniques that come close to users and their rich experiences might have value for pervasive-healthcare applications. Much more effort is needed

\section{IEEE Intelligent Systems delivers} the latest peer-reviewed research on all aspects of artificial intelligence, focusing on practical, fielded applications. Contributors include leading experts in

- Intelligent Agents • The Semantic Web - Natural Language Processing

- Robotics • Machine Learning

Visit us on the Web at www.computer.org/intelligent
Mieke van Gils is a researcher at Katholieke Universiteit Leuven's Center for Usability Research. Contact her at mieke.vangils@soc. kuleuven.be.

Jan-Willem van 't Klooster is a PhD research assistant at the University of Twente. Contact him at j.w.vantklooster@utwente.nl.

Annelies Veys is a researcher at the Center for Studies on Media, Information and Telecommunication (SMIT) at the Interdisciplinary Institute for Broadband Technology. Contact her at annelies.veys@vub.ac.be.

Mohammed Zarifi Eslami is a PhD research assistant at the University of Twente. Contact him atm.zarifi@ewi.utwente.nl.

to include specific target groups in the design of these applications. Although best practices regarding user involvement, usability, and acceptance of pervasive applications are at hand, current design methods are rarely helpful in guiding designers to incorporate human values. The workshop shows that researchers are now actively looking for ways to incorporate human values in the design of pervasive-health applications, and based on their experiences in these design cases, designers of future pervasive-healthcare applications can better incorporate user values in their designs. P

\section{REFERENCES}

1. V. Venkatesh et al., "User Acceptance of Information Technology: Toward a Unified View," Management Information Systems Quarterly, vol. 27, no. 3, 2003, pp. 425-478.

2. T. Sohn, "A Diary Study of Mobile Information Needs," Proc. 26th Ann. SIGCHI Conf. Human Factors in Com puting Systems (CHI 08), ACM Press, 2008, pp. 433-442. 